\title{
Revealing the Working Active Sites of M1 phase for Ethane Oxidation
}

Yuanyuan Zhu ${ }^{1}$, Eric Jensen ${ }^{1}$, Peter V. Sushko ${ }^{1}$, Libor Kovarik ${ }^{2}$, Daniel Melzer ${ }^{3}$, Maricruz SanchezSanchez ${ }^{3}$, Johannes A. Lercher ${ }^{3}$ and Nigel D. Browning ${ }^{1,4}$

1. Physical \& Computational Sciences Directorate, Pacific Northwest National Laboratory, Richland, WA 99352, USA

2. Environmental Molecular Sciences Laboratory and Institute for Integrated Catalysis, Pacific Northwest National Laboratory, Richland, WA 99352, USA

3. Department of Chemistry and Catalysis Research Center, Technical University of Munich, Garching 85748, Germany

4. Department of Materials Science and Engineering, University of Washington, Seattle, WA 98195 , USA

At the heart of understanding heterogeneous catalysis lays the identification of the nature and structure of active sites - an ensemble of atoms new pathways for the desired reactions. Active sites facilitate reactant/product molecules absorption/desorption, bond rearrangement and electron exchange, but active sites often undergo themselves geometric and electronic structural changes under working conditions. In the field of oxidative dehydrogenation (ODH) of alkanes, understanding the catalytically relevant sites in the most promising catalyst - a complex MoVNbTe oxide in its crystalline form designated as M1 phase - remains as one of the greatest challenges $[1,2]$. Among various structural characterization tools, environmental annular dark-field scanning transmission electron microscopy (ADF-STEM) offers unique directly interpretable incoherent imaging of potential catalytic sites at the atomic level [3, 4]. In this work, we demonstrate systematic ADF-STEM analysis of the atomic configuration of the M1 (001) basal plane, where active sites are proposed to be located, after quenching from the ethane ODH operation temperature of $400{ }^{\circ} \mathrm{C}$ in the presence of various gas feeds.

Fig. 1 summarizes the structural evolution of the same M1 (001) at various heating durations under each carefully chosen atmospheric condition including: 1) (S)TEM vacuum $\left(\sim 10^{-8} \mathrm{mbar}\right.$, an unwanted highvacuum required by conventional (S)TEM as used in [5]), 2) inert He of 0.9 mbar (to bridge the pressure gap), 3) pure oxidative atmosphere with an oxygen partial pressure of 0.9 mbar and 4) the ethane ODH reaction atmosphere. Here, we employed a dedicated field-emission ETEM (FEI Titan 80-300), equipped with differential pumping system allowing controlled gas pressure around the sample, and a resistant coil double-tilt Gatan heating holder. To alleviate heating-introduced sample drifting and offzone, we adopted a slow-heating-and-fast-quenching protocol that preserves the development of M1 structure at different reaction stages for atomic STEM observation.

To achieve a sound sampling statistics, we developed an automated line profile analysis capable of robust locating and effectively subtracting intensity and atomic displacement information over 200 studied structure units (marked by diamonds) for each STEM image. This in-depth analysis reveals unexpected structural modifications of atomic sites round the cation site S2 in the M1 (001) plane when exposed to different gas feeds at different heating duration. Combining STEM image quantification and simulation as well as ab initio (density functional theory) simulations of the temperature-induced transformations of the atomic structure and associated electronic structure changes, we propose a new model for the M1 active sites under working conditions for ethane oxidation as well as for the evolution of such active sites from the room-temperature stable catalyst precursor. 


\section{References:}

[1] R Schlögl, Topics in Catalysis 54 (2011) 627.

[2] C Gaertner, A van Veen and J Lercher Chemcatchem 5(2013) 3196.

[3] E Boyes and P Gai, Comptes Rendus Physique 15 (2014) 200.

[4] N Browning et al, Chemcatchem 5(2013) 2673.

[5] D Blom et al, Topics in Catalysis 57 (2014) 1138.

[6] This research is part of the Chemical Imaging Initiative conducted under the Laboratory Directed Research and Development Program at Pacific Northwest National Laboratory (PNNL). PNNL, a multiprogram national laboratory, is operated by Battelle for the Department of Energy under Contract DE-AC05-76RLO1830.



Figure 1. Atomic resolution ADF-STEM snapshots of M1 (001) planes before and after thermally treated for different heating durations in four systematically selected atmospheric conditions. The inserts in the $1^{\text {st }}$ row are simulated STEM images from M1 crystal with modified S12-Te(O) occupancy and atomic displacement. 